\title{
Application of New Generation Geometrical Product Specifications in the practice in Small and Medium Sized enterprises
}

\author{
Pavlina Toteva ${ }^{1, *}$ and Krasimira Koleva ${ }^{l}$ \\ ${ }^{1}$ Technical University of Varna, 1 Studentska str., pavlina_toteva@abv.bg
}

\begin{abstract}
The increasing development of modern methods of product manufacturing implies an improvement and a new approach to the modern drawing specification in design documentation for design requirements of different parts and assemblies. The ISO Technical Committee 213 has published 148 standards and 48 other standards are under development. These standards set out requirements for the accuracy of geometrical characteristics of products. They define requirements for the linear sizes, dimensions different than linear sizes as well as geometric deviations such as form, orientation, location deviations and run-out, and surface roughness and waviness. Ways of indication of these requirements in the drawings are discussed. Many standards are dedicated to the requirements for measuring these characteristics, to measuring instruments for linear sizes and geometrical characteristics. This report reviews the general concept of geometrical product specifications (GPSs), the GPS model, and new terminology by tolerancing linear sizes, geometrical specifications that facilitate communication between design manufacturing and verification and prevent ambiguous interpretation of requirements. Based on the metrological expertise of the drawings in a number of small and mediumsized enterprises, the degree of implementation of the new generation GPS standards and the problems related to it have been analysed.
\end{abstract}

\section{Introduction}

Globalization is progressing in all areas of manufacturing. Designers, manufacturers, customers and suppliers are often located in different countries. In these cases, employees of design, manufacturing and quality assurance have no direct contact with each other. It is therefore all the more important to use international drafting standards for a technical drawing - only in this way can misinterpretations be ruled out.

The basic standards of the new generation of Geometrical Product Specifications (GPS) that must be known and applied in drawings, in manufacturing and in inspection are related to the requirements for accuracy of linear and angular sizes according to ISO 286-1[1], ISO 14405-1[2] and ISO 14405-3[3], the requirements of dimensions other than linear sizes according to ISO 14405-2[4], the requirements of general tolerances for linear and angular

*Corresponding author: pavlina toteva@abv.bg 
dimensions without individual tolerance indications according to ISO 2768-1 [5] and the geometrical tolerances for features without individual tolerance indications according to ISO 2768-2[6], the requirements of geometrical tolerancing of form, orientation, location, and run-out according to ISO 1101[7], the functional bevels and defined transitions between features according to ISO 1660[8], the requirements of non-functional edges, bevels and rounding according to ISO 13715 [9], and the requirements of surface roughness according to ISO 1302 [10].In order to introduce these standards into practice, it is necessary to study their real implementation and the gaps in the drawings, and to identify operations for their prevention.

\section{The General concept of GPS tolerancing}

The purpose of each production is to ensure flawless functioning, safety, reliability and interchangeability of the products. This is achieved by applying the GPS standards..

The ISO GPS standard system (GPS = Geometrical Product Specification) is composed of large number of standards to provide consistent rules ("tools") allowing full description of geometrical features related to size, shape, direction, location or run-out in technical specifications. Furthermore, the standards include rules for a clear proof of conformity of the finished product with the specified requirements (verification). Another set of standards includes the associated measuring equipment, the calibration procedure for these measuring instruments as well as the measurement uncertainty.

As explained in the introduction of ISO/FDIS 22432:2011(E) [11] Geometrical features exist in three "worlds": nominal model which is created in CAD system on the drawing, the skin model created by designer and the real model as result of manufacturing.

At the end of ISO GPS's operational implementation process, there are clear and functionoriented product specifications in a geometric "language" that can be understood worldwide for development, production and measurement. This language must be interpreted unequivocally at all stages of the manufacturing of products as shown in Figure 1.

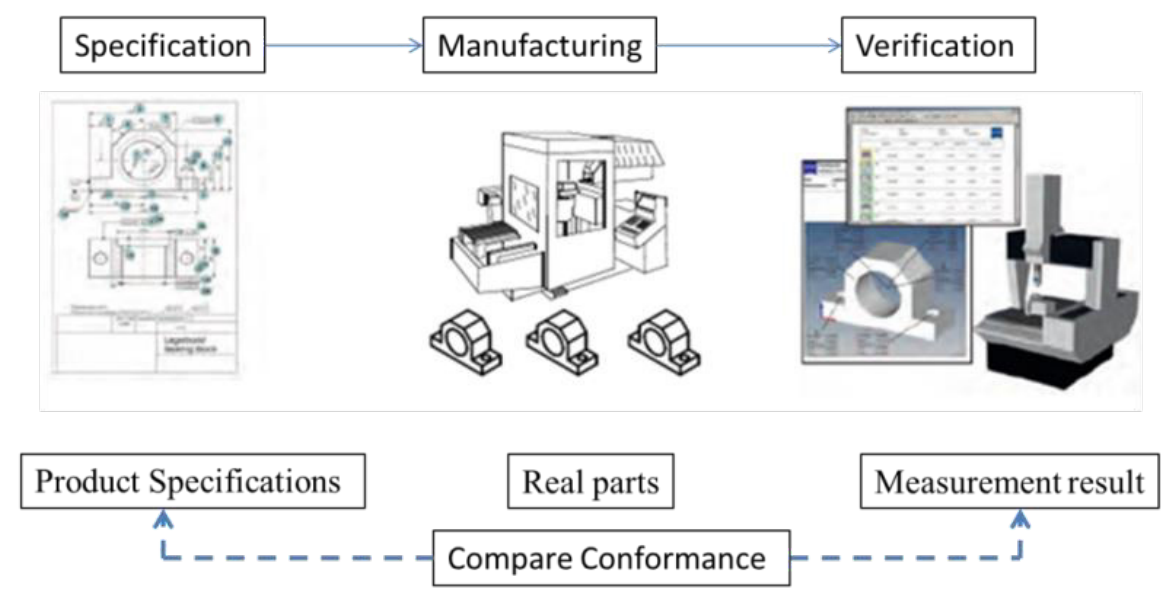

Fig.1. Communication between design manufacturing and verification

The implementation of the GPS standards' concepts has an impact on the fitting, function, safety and quality of products. (see Figure 2). 


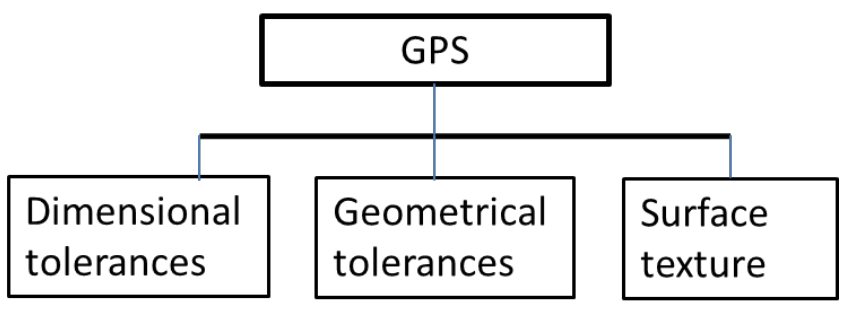

Fig.2. Tolerancing according GPS concept

Figure 3 shows the elements included within GPS models.

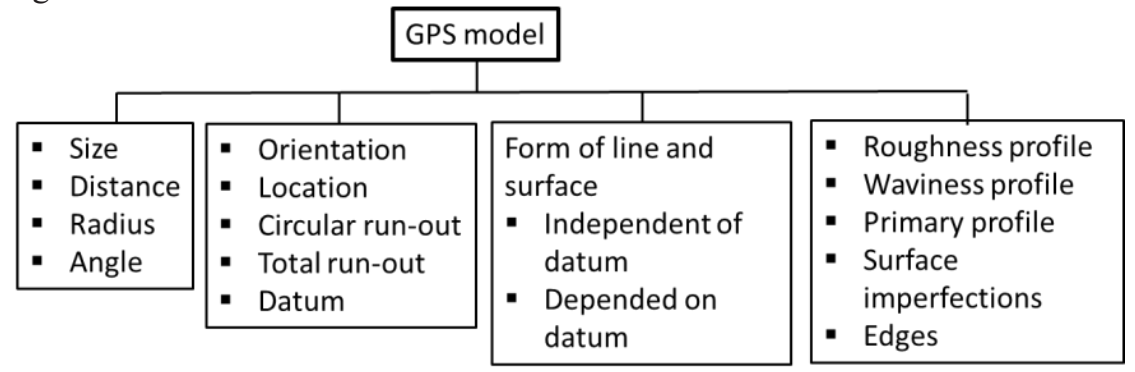

Fig. 3. The GPS tolerancing model

\subsection{Dimensional tolerances}

Figure 4 shows the types of dimensions. The tolerances for linear sizes, external and internal linear sizes respectively (Fig. 4a and 4b), are described in ISO 286-1[1] and the types of linear sizes in ISO 14405-1[2]. (see Figure 5)

a)
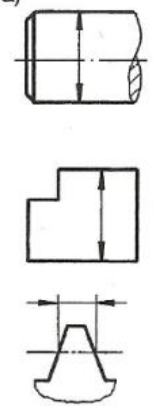

b)
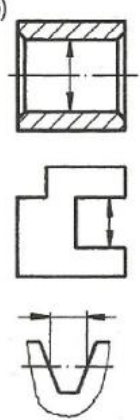
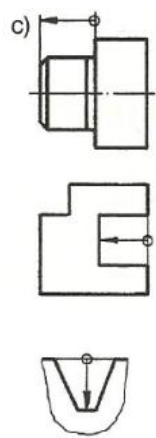

d)
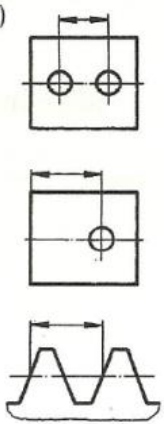

Fig.4: Dimensioning groups: a) external dimensions, b) internal dimensions, c) step dimensions, d) distance dimensions [12]

Dimensions other than linear sizes are toleranced according to the rules in ISO 14405-2 [4] and refer to geometrical tolerances. 


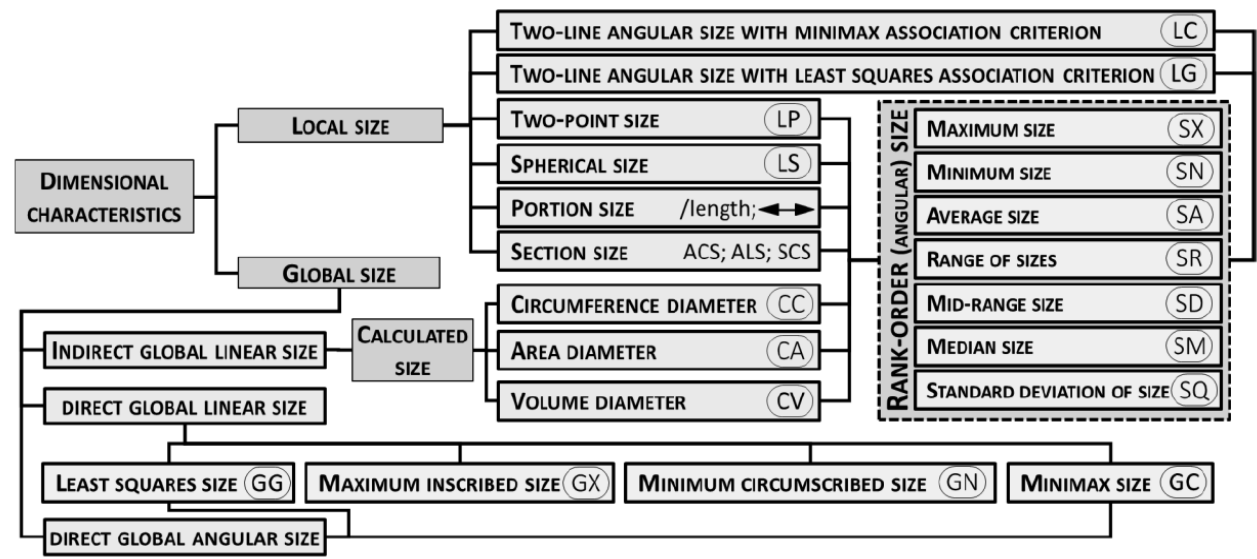

Fig. 5. Specification modifiers for linear and angular sizes [13].

\subsection{Geometrical tolerances}

The basic standards with the types of geometric deviations are ISO 1101, ISO 1660 ISO 5459 , ISO $5458[7,8,14,15]$ and can be grouped into three groups as shown in Figure 3.

Table 1. Geometrical tolerances and tolerance symbols[16]

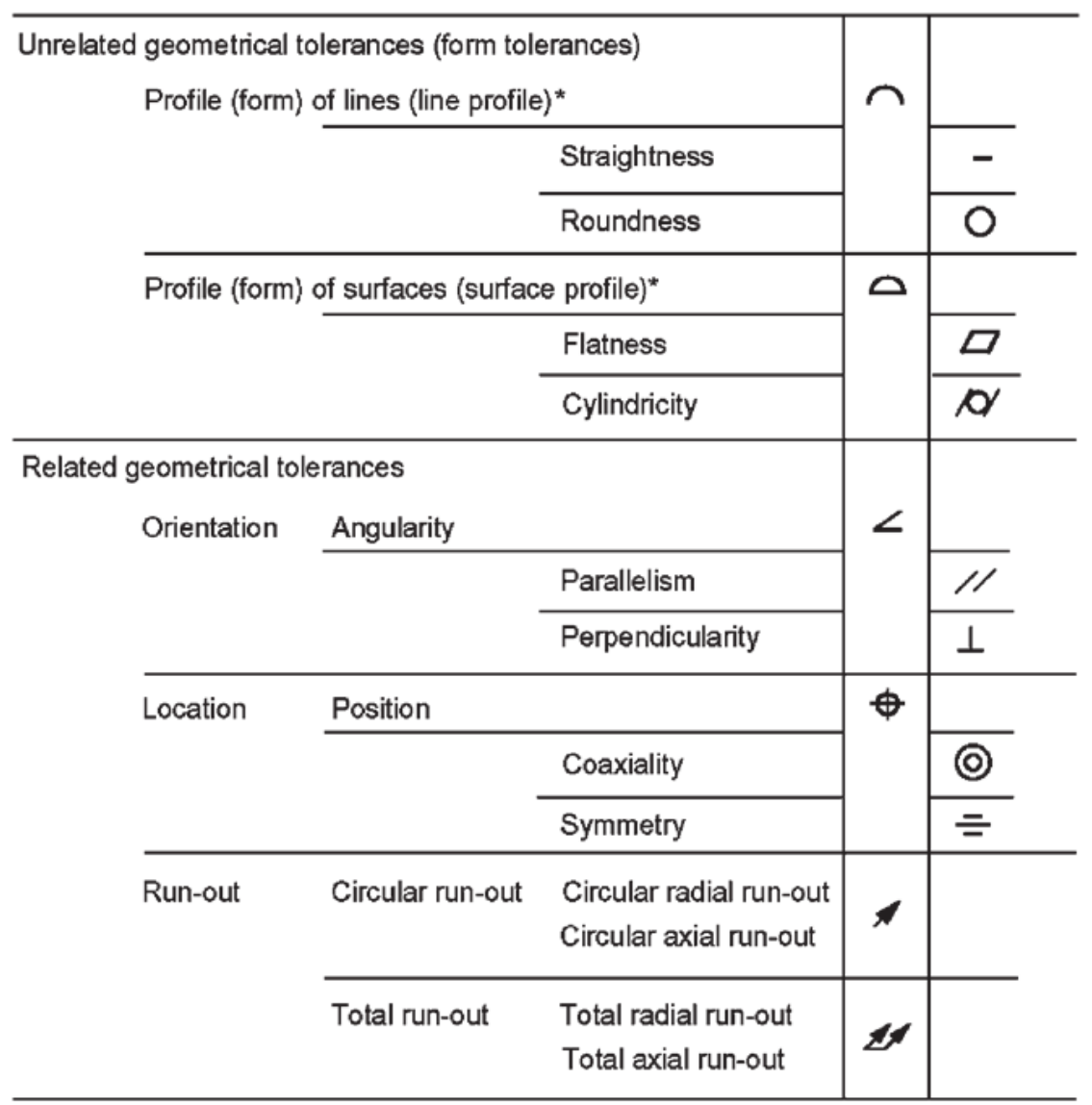


Along with the set of symbols for geometrical tolerances in this standard many additional symbols and modifiers are described as shown in tables 2, 3 and 4 .

Table 2. ISO special specification elements for form-parameter symbols.

\begin{tabular}{|c|l|}
\hline Symbol & \multicolumn{1}{c|}{ Parameter } \\
\hline $\mathrm{P}$ & Reference-to-peak \\
\hline $\mathrm{V}$ & Reference-to-valley \\
\hline $\mathrm{T}$ & Peak-to-valley \\
\hline $\mathrm{Q}$ & Root mean square (RMS) \\
\hline
\end{tabular}

Table 3. ISO special specification elements for form-association symbols

\begin{tabular}{|c|c|c|}
\hline Symbol & \multicolumn{2}{|c|}{$\begin{array}{c}\text { Association Operation } \\
\text { (Objective functions and constraints) }\end{array}$} \\
\hline $\mathrm{C}$ & Minimax (Chebyshev) criterion & \multirow[t]{2}{*}{ Objective function } \\
\hline G & Least-squares (Gaussian) criterion & \\
\hline $\mathrm{x}$ & Maximum inscribed criterion & \multirow{2}{*}{$\begin{array}{l}\text { Objective function } \\
\text { and constraint }\end{array}$} \\
\hline $\mathrm{N}$ & Minimum circumscribed criterion & \\
\hline$E$ & Constrained external to the material & \multirow[t]{2}{*}{ Constraint } \\
\hline I & Constrained internal to the material & \\
\hline
\end{tabular}

Table 4. Symbols for association operation on toleranced features

\begin{tabular}{|c|c|}
\hline Symbol & Association Operation \\
\hline (c) & Chebyshev (minimax) \\
\hline () & Gaussian (least-squares) \\
\hline (i) & Minimum circumscribed \\
\hline (1) & Tangent: Constrained least-squares \\
\hline$\otimes$ & Maximum inscribed \\
\hline
\end{tabular}

\subsection{Geometrical references (datum features)}

ISO 5459 specifies terminology, rules and methodology for the indication and understanding of datums and datum systems in technical product documentation. This International Standard also provides explanations to assist the user in understanding the concepts involved. In this standard, the definition and realization by measuring the common datums as shown in Fig.6 is important.

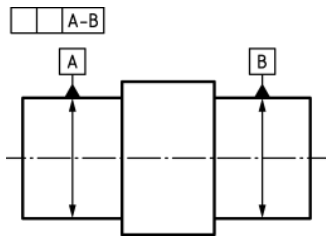

a.

b.
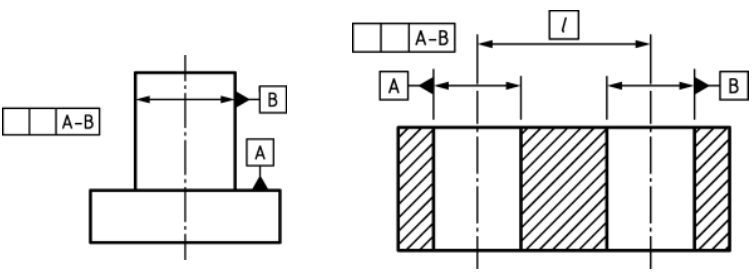

c. 


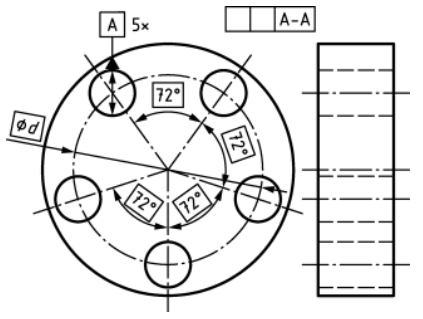

d.

a- common datum established from two coaxial cylinders

b- common datum established from a plane and a perpendicular cylinder

c- common datum established from two parallel cylinders

d- common datum established from a pattern of five cylinders

e- common datum established from two parallel planes

f- common datum established from intersecting planes

Fig.6 Common datum:

\subsection{Tolerancing principles}

ISO 8015(17) specifies fundamental concepts, principles, and rules valid for the creation, interpretation and application of all other International Standards, Technical Specifications and Technical Reports concerning dimensional and geometrical product specifications (GPS) and verification. 13 principles are provided by the standard. Figure 7 shows the most important of them

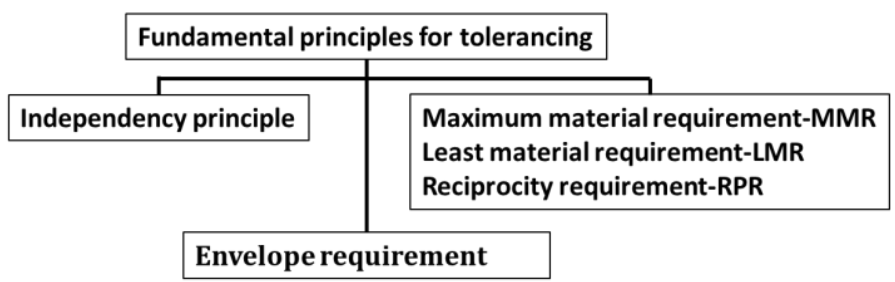

Fig.7 Principles of tolerancing

Principles that are not marked on the drawings but always apply by default are: the rectangular coordinate system is used in the drawings, independency principle, the material condition default is free, the measuring temperature is always $200 \mathrm{C}$, the dimensions of the linear sizes are millimeters, the roughness requirements are set in micrometers, and angular dimensions - in degrees, minutes and seconds.

\section{Analysis of the requirements depicted in the drawings according to the new generation of GPS standards}

Overview of the requirements in the drawings of several middle and small enterprises, according to requirements of GPS standards, is outlined as follows:

- Designation requirements for accuracy of linear and angular sizes according to ISO 286-1, ISO 14405-1 and ISO 14405-3.

Comment: The tolerance values depicted in the drawings do not match to the standards' values provided by standard quality in ISO 286-1.

Operators for the types of functional linear sizes, according to ISO 14405-1 as local (LP, LS), global (GG, GX, GN, GC), specified depending on the function of the workpiece, can not be found in drawings. 
- Designation requirements of dimensions other than linear sizes according to ISO 14405-2.

Comment: The requirements of this standard are not met, therefore the standard is not known.

- Designation requirements of general tolerances for linear and angular dimensions without individual tolerance indications according to ISO 2768-1 [5] and geometrical tolerances for features without individual tolerance indications according to ISO 2768-2 [6].

Comment: All non-functional sizes in the drawings are tolerated with a tolerance zone (Js13 or H14). The tolerances of these dimensions and geometrical tolerances are not designated according to ISO $2768-1$ and ISO $2768-2$. In $90 \%$ of the drawings general tolerances of geometrical tolerances are not included.

- Designation requirements of geometrical tolerancing form, orientation, location, and run-out and according to ISO 1101.

These requirements in drawings are designated only with symbols depicted in Table 1. Modifier symbols depicted in Tables 2, 3 and 4, which depended on the function of the details and determine how to measure and assess the comfortability, are not depicted in the drawings. In $90 \%$ of the drawings general tolerances of geometrical deviations are not included.

- Functional bevels and defined transitions between features according to ISO 1660 Comment: The requirements of standards are not met.

- Designation requirements of non-functional edges, bevels and roundings according to ISO 13715 [9].

Comment: The requirements of standards for non-functional edges, bevels and rounding's rounds do not meet the requirements according to ISO 13715 (short, clear and visually).

- Designation requirements of surface roughness according to ISO 1302 [18].

Comment: In $50 \%$ of the drawings, roughness does not meet the standard requirements.

To eliminate the gaps in the construction documentation, underlying factors and reasons have to be analyzed. Among these are:

- A large number of standards, with a huge amount of information;

- Many new characters and modifiers for requirements' indication;

- In companies, the introduction of GPS standards is assumed to cause high costs;

- In companies, the introduction of GPS standards is assumed to be appropriate for mass and serial production;

- The designers suggest that working with fewer geometrical requirements with acceptable results can be continued;

- The requirements of GPS standards are very complex and difficult to understand;

- For some of the geometrical accuracy requirements, modern measuring instruments, unavailable in small- and medium-sized enterprises, are required

\section{Conclusions}

ISO GPS is a "geometrical language" which defines the symbolism for all relevant geometrical features, tolerance features, characteristics of the associated geometrical elements on the finished workpiece, and requirements for the comparison between measurement results and specification.

The advantages of using ISO GPS standards are:

- Function dependent, unique and complete specification

- No more (hidden) specifications, which are not described in the drawing 
- Minimize liability risks by means of a functional, production-, test- and cost-oriented geometrical toleration

- Increase the quality of the products

- Less waste due to clear specifications and verifications

- Globalization: no necessity for design, manufacturing and quality assurance to be in the same place

- Digitization of production and measuring systems and thus reduction of development times and testing effort

- Reduce costs

\section{References}

1. ISO 286-1:2010(en), Geometrical product specifications (GPS) — ISO code system for tolerances on linear sizes - Part 1: Basis of tolerances, deviations and fits

2. ISO 14405-1:2016, Geometrical product specifications (GPS) -- Dimensional tolerancing -- Part 1: Linear sizes

3. ISO 14405-3:2016, Geometrical product specifications (GPS) - Dimensional tolerancing - Part 3: Angular sizes

4. ISO 14405-2:2011, Geometrical product specifications (GPS) - Dimensional tolerancing —-Part 2:Dimensions other than linear sizes

5. ISO 2768-1:1989, General tolerances - Part 1: Tolerances for linear and angular dimensions

6. ISO 2768-2:1989, General tolerances - Part 2: Geometrical tolerances for features without individual tolerance indications

7. ISO 1101:2017, Geometrical product specifications (GPS) - Geometrical tolerancing - Tolerances of form, orientation, location and run-out

8. ISO 1660:2017, Geometrical product specifications (GPS) - Geometrical tolerancing - Profile tolerancing

9. ISO 13715:2017(en), Technical product documentation - Edges of undefined shape - Indication and dimensioning

10. ISO 1302:2002 Geometrical Product Specifications (GPS) -- Indication of surface texture in technical product documentation

11. ISO/FDIS 22432:2011, Geometrical product specifications (GPS) -- Features utilized in specification and verification

12. W. Jorden,: Form- und Lagetoleranzen - Handbuch für Studium und Praxis 3.Auflage. Carl Hanser Verlag, München / Wien, 2005

13. P.Karel, 58th ICMD 2017, 6 - 8 September, Prague, Czech Republic (2017)

14. ISO/DIS 5459:2016 Geometrical product specifications (GPS) - Geometrical tolerancing - Datums and datum systems

15. ISO 5458:2018 Geometrical product specifications (GPS) -- Geometrical tolerancing -Pattern and combined geometrical specification

16. Henzold G. Geometrical Dimensioning and Tolerancing for Design, Manufacturing and Inspection 2nd Edition, A Handbook for Geometrical Product Specification using ISO and ASME standards,2006, Butterworth-Heinemann

17. ISO 8015:2011, Geometrical product specifications (GPS) - Fundamentals Concepts, principles and rules 